Document downloaded from:

http://hdl.handle.net/10251/142037

This paper must be cited as:

Ballester-Bolinches, A.; Pedraza Aguilera, MC. (02-2). On a theorem of Kang and Liu on factorised groups. Bulletin of the Australian Mathematical Society. 97(1):54-56. https://doi.org/10.1017/S0004972717000363

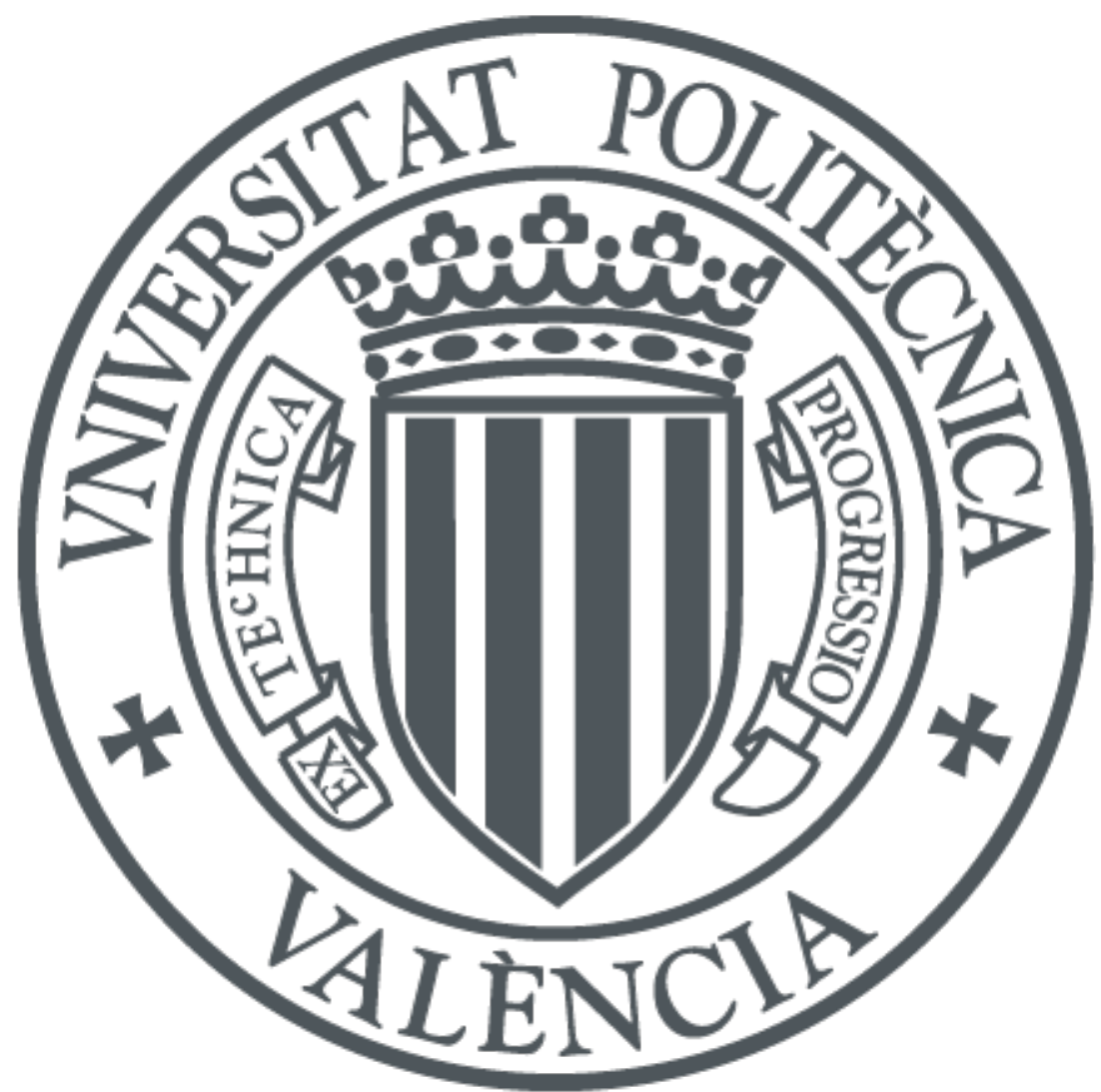

The final publication is available at

https://doi.org/10.1017/S0004972717000363

Copyright Cambridge University Press

Additional Information 


\title{
On a theorem of Kang and Liu on factorised groups
}

A. Ballester-Bolinches and M.C. Pedraza-Aguilera

\begin{abstract}
Kang and Liu ['On supersolvability of factorized finite groups', Bull. Math. Sci. 3 (2013), 205-210] investigate the structure of finite groups that are products of two supersoluble groups. The goal of this note is to give a correct proof of their main theorem.

Mathematics Subject Classification (2010): 20D10, 20D20, 20D40

Keywords: finite group, factorisations, supersolubility.
\end{abstract}

\section{Introduction}

All groups considered in this paper are finite.

We recall that two subgroups $A$ and $B$ of a group $G$ are said to permute if $A B$ is a subgroup of $G$. Further, $A$ and $B$ are called mutually permutable if every subgroup of $A$ permutes with $B$ and every subgroup of $B$ permutes with $A$.

Products of mutually permutable subgroups have been widely studied in the last twenty-five years and receive a full discussion in [3]. The emphasis is on how the structure of the factors $A$ and $B$ affects the structure of the factorised group $G=A B$ and vice versa.

The goal of the present paper is to give a correct proof of the main result of the paper [5]. Therefore this paper had best be read in conjunction with [5].

First we recall the main theorem of that paper.

Theorem A ([5, Theorem C]). Let the group $G=H K$ be the product of the subgroups $H$ and $K$. Assume that $H$ permutes with every maximal subgroup of $K$ and $K$ permutes with every maximal subgroup of $H$. If $H$ is supersoluble, $K$ is nilpotent and $K$ is $\delta$-permutable in $H$, where $\delta$ is a complete set of Sylow subgroups of $H$, then $G$ is supersoluble. 
The statement of the Theorem A resembles that of a theorem of Cossey and the authors [2, Theorem 3], which asserts the same conclusion under the stronger assumption that $K$ permutes with every Sylow subgroup of $H$. The proof of Theorem A presented in [5] is not new, but it is an exact copy of the proof of [2, Theorem 3]. However, it is abundantly clear that this proof does not hold if $K$ is $\delta$-permutable in $H$. In fact, in the last paragraph of the proof (as in [2, Theorem 3]), the authors wrote:

"By hypothesis, $K$ is $\delta$-permutable in $H$, where $\delta$ is a complete set of Sylow subgroups of $H$. Hence we can easily deduce that $K$ permutes with every Hall $p^{\prime}$-subgroup of $H^{\prime \prime}$.

There are many examples showing that this claim is false in general (consider, for example, the symmetric group of degree four which is a product of the alternating group and a transposition). We need $K$ to be permutable with all Sylow subgroups of $H$ to ensure that $K$ permutes with every Hall $p^{\prime}$-subgroup of $H$.

\section{Proof of Theorem A}

Proof. Assume the result is false and let $G$ be a counterexample of minimal order. By [2, Theorem 1], $G$ is soluble. Let $1 \neq N$ be a normal subgroup of $G$. It is clear that the hypotheses of the theorem hold in $G / N$. By minimality of $G, G / N$ is supersoluble. Consequently, $G$ has a unique minimal normal subgroup $N$ which is abelian and complemented in $G$ by a core-free maximal subgroup $M$ of $G$. Let $p$ be the prime dividing $|N|$ and let $q$ be the largest prime dividing $|G|$.

Assume that $p \neq q$. Let $H_{q}$ be a Sylow $q$-subgroup of $H$. Then $H_{q}$ is a normal subgroup of $H$ because $H$ is supersoluble. Moreover $K$ has a unique Sylow $q$-subgroup because $K$ is nilpotent. Applying [1, Lemma 2.4.2], we see that $H_{q}$ permutes with $K_{q}^{g}$ for each $g \in G$. Since $O_{q}(G)=1$, it follows that $\left[H_{q}{ }^{G}, K_{q}{ }^{G}\right]=1$ by [1, Lemma 2.5.1]. It is quite clear that we can assume that either $H_{q}{ }^{G} \neq 1$ or $K_{q}{ }^{G} \neq 1$ because, otherwise, $G$ would be a $q^{\prime}$-group.

Suppose that $H_{q}{ }^{G} \neq 1$ (the case $K_{q}{ }^{G} \neq 1$ is analogous). Then $N$ is contained in $H_{q}{ }^{G}$. Therefore $\left[N, K_{q}{ }^{G}\right]=1$ and $K_{q}{ }^{G} \leq C_{G}(N)=N$. Hence $K_{q}{ }^{G}=1$ and $K$ is a $q^{\prime}$-group. Since every Sylow $q$-subgroup of $M$ is a Sylow $q$-subgroup of $G$, we may assume that $H_{q}$ is contained in $M$. Since $M$ is supersoluble, it follows that $H_{q}$ is normalised by $M$. If $G=N_{G}\left(H_{q}\right)$, then $N$ is contained in $H_{q}$, which is a contradiction. Thus $M=N_{G}\left(H_{q}\right)$. This implies that $H$ is contained in $M$. Therefore $M=H(M \cap K)$. Hence $M \cap K$ is a maximal subgroup of $K$. Applying [4, Lemma 2.3], we deduce that $K$ is a Sylow $q$-subgroup of $G$ with $|K|=q$. Moreover $H=M$ and $|G: H|=q$. 
Then $N \leq M$, which is a contradiction.

Suppose now that $p$ is the largest prime dividing $|G|$. Since $M$ is supersoluble and $O_{p}(M)=1$, we see that $M$ is a $p^{\prime}$-group and so $N$ is a Sylow $p$-subgroup of $G$. In particular $G$ is a Sylow tower group of supersoluble type. Let $K_{p^{\prime}}$ be the Hall $p^{\prime}$-subgroup of $K$. Assume that $(H \cap K) K_{p^{\prime}}$ is a proper subgroup of $K$ and let $K_{0}$ be a maximal subgroup of $K$ containing $(H \cap K) K_{p^{\prime}}$. Then $H K_{0}$ is a proper subgroup of $G$. Write $S=H K_{0}$. If $\operatorname{Core}_{G}(S)=1$, then $S \cap N=1$ and $G=S N$. Hence $|N|=|G: S|=\left|H K: H K_{0}\right|=\left|K: K_{0}\right|=p$, which is a contradiction. Suppose that $\operatorname{Core}_{G}(S) \neq 1$. Then $N$ is contained in $H K_{0}$ and so $N$ is a Sylow $p$-subgroup of $H K_{0}$. Since $H \cap K=H \cap K_{0}$, it follows that $K_{0}$ contains a Sylow $p$-subgroup of $K$. This contradiction shows that $K=(H \cap K) K_{p^{\prime}}$ and so $N$ is contained in $H$. In particular, $H=N(H \cap M)$.

Let $q \neq p$ be a prime and let $H_{q}$ be a Sylow $q$-subgroup of $H$ permuting with $K$. Then $X=K H_{q}=(H \cap K) K_{p^{\prime}} H_{q}$ is a subgroup of $G$. Since $X$ is a Sylow tower group of supersoluble type, it follows that the Sylow $p$-subgroup $A$ of $H \cap K$ is normal in $X$. Hence $A$ is normalised by $H_{q}$. This implies that $A$ is a normal subgroup of $H$ and so $A$ is normal in $G$. Consequently $A=N$ or $A=1$. Assume that $A=N$ so that $K=N K_{p^{\prime}}$. Let $N_{1}$ denote a minimal normal subgroup of $H$ with $N_{1} \leq N$. Then $\left|N_{1}\right|=p$ and $K$ normalises $N_{1}$. Therefore $N=N_{1}$, which is a contradiction. Thus we may assume that $A=1$. In this case, $K$ is contained in $M, M=K(M \cap H)$ and $M \cap H$ is a maximal subgroup of $H$. Therefore $p=|H: M \cap H|=|N|$, which is the final contradiction.

\section{References}

[1] B. Amberg, S. Franciosi and F. De Giovanni, Products of Groups, Oxford Mathematical Monographs, Clarendon Press, Oxford 1992.

[2] A. Ballester-Bolinches, John Cossey and M.C. Pedraza-Aguilera, On products of finite supersoluble groups, Comm. in Algebra, 29(7), 31453152, 2001.

[3] A. Ballester-Bolinches, R. Esteban-Romero and M. Asaad, Products of finite groups, vol. 53 of the Gruyter Expositions in Mathematics. Walter de Gruyter, Berlin 2010.

[4] L. M. Ezquerro and X. Soler-Escrivà, On mutually m-permutable products of finite groups, Comm. in Algebra, 31(4), 1949-1960, 2003. 
[5] Ping Kang and Qingfeng Liu, On supersolvability of factorized finite groups, Bull. Math. Sci., 3, 205-210, 2013.

A. Ballester-Bolinches

Departament de Matemàtiques, Universitat de València

Dr. Moliner 50, 46100 Burjassot, València (Spain)

e-mail: Adolfo.Ballester@uv.es

M.C. Pedraza-Aguilera

Instituto Universitario de Matemática Pura y Aplicada

Universitat Politècnica de València,

Camino de Vera, 46022, Valencia (Spain)

e-mail: mpedraza@mat.upv.es 